\title{
Cobro de servicios aeroportuarios a aeronaves privadas en el Aeropuerto Internacional Toncontín
}

Alex Matamoros, José Orlando Ramos

\section{Resumen}

Los cobros por servicios aeroportuarios en el Aeropuerto Internacional Toncontín, se derivan de la aplicación de la Ley de Aeronáutica Civil y del contrato de Concesión que el Estado tiene con la Empresa Interairport; el hacer efectivos estos cobros corresponde a la Concesionaria en coordinación con la Agencia Hondureña de Aeronáutica Civil. Este artículo analiza las razones por las cuáles estos cobros no se dan con un cien por ciento de efectividad. La intención de fondo del análisis es descubrir los obstáculos que están disminuyendo el porcentaje de cobros y que pueden obstruir otros cobros y otras coordinaciones entre la Concesionaria y la Agencia Hondureña de Aeronáutica Civil (AHAC), que se deben hacer en condiciones similares.

Palabras clave: Cobros, Aeronaves privadas, contrato de concesión, control.

\section{Abstract}

Charges for airport services are derived from the application of the Law of Civil Aeronautics and concession contract that the state has to Interairport Company; the make effective these charges corresponds to the Concessionaire in coordination with the Honduran Civil Aviation Agency. This article analyzes the reasons why these charges do not give a hundred percent effective. The intention behind the analysis is to discover the obstacles that are decreasing the percentage of charges and other charges that can obstruct and other coordination between the Concessionaire and HCAA, which must be done under similar conditions.

Keywords: Collections, private aircraft, concession contract control. 
Alex Matamoros, (algemaca@hotmail.com) Departamento de Ciencias Aeronáuticas, Facultad de Ciencias Espaciales, Universidad Nacional Autónoma de Honduras - UNAH, José Orlando Ramos, (orlando.ramos@popeyes.hn)Investigador Colaborador Departamento de Ciencias Aeronáuticas 


\section{Introducción}

Una de las razones principales por las que se tiene una reglamentación de las operaciones aeroportuarias es el incentivo de la industria aeronáutica, sin que ello afecte negativamente a los usuarios de servicios aeroportuarios, ni la eficiencia en la prestación de servicios de las autoridades aeroportuarias. En el centro de este conjunto de actores aeronáuticos están por una parte los aeropuertos y los proveedores de servicios de navegación aérea, por otra, los transportistas aéreos; al Estado en su función reguladora le corresponde alentar una mayor cooperación entre los actores mencionados de forma que todos compartan de manera razonable la carga de las dificultades económicas que deben afrontar .

En un primero bloque de la investigación se explica que su objetivo es identificar las razones por las cuales los pilotos privados no hacen los pagos correspondientes por servicios aeroportuarios en el Aeropuerto Internacional Toncontín, en el entendido que en otros aeropuertos del país se da una situación similar y previendo que las propuestas de solución a los obstáculos detectados pueden ser útiles para mejorar otras coordinaciones que se dan en la esfera de relaciones entre el Estado de Honduras y la Consecionaria Interairport. En un segundo bloque, se explica la metodología utilizada, la cual propone una perspectiva indagatoria en la que se privilegia la consulta a pilotos privados y representantes de la Autoridad aeronáutica hondureña; además, parte de la metodología aplicada fue el contraste de las opiniones recogidas, con el marco reglamentario que establece el contrato de concesión y las estadísticas disponibles. Un tercer bloque de la investigación lo constituyen tres resultados: un resumen de las tarifas y cobros establecidos relacionados con el tema estudiado; el procedimiento de cobro por servicios aeroportuarios vigente en Toncontín por servicios aeroportuarios a aeronaves privadas; los resultados de las consultas con los usuarios de los servicios mencionados; cabe mencionar que en cada gráfico de resumen de resultados se hace un análisis de los datos obtenidos. El último bloque lo constituyen las conclusiones y recomendaciones.

Se espera que la importancia del presente trabajo sea dada no tanto por los montos que se dejan de cobrar por servicios aeroportuarios prestados a aeronaves privadas, sino más bien por el examen hecho de la coordinación de la Concesionaria con el Estado de Honduras; puesto que una buena coordinación para hacer esta recaudación puede producir lecciones aprendidas que mejoren otros procesos con mayor impacto económico para la aeronáutica civil hondureña. 


\section{Objetivos}

\section{Objetivo General}

Identificar cuál ha sido la causa por la cual los pilotos de vuelos privados no cumplen con el procedimiento de pago de servicios aeroportuarios al concesionario Aeropuertos de Honduras antes de la aprobación del plan de vuelo y del despegue de la aeronave del aeropuerto Internacional Toncontín.

\section{Objetivos específicos}

- Investigar cuál es el procedimiento que actualmente están siguiendo los pilotos de vuelos privados al ingresar y al salir del aeropuerto.

- Verificar el conocimiento que los pilotos de vuelos privados tienen del procedimiento de pago por servicios e indagar las razones por las que no se ha cumplido dichos pagos.

- Sugerir algunas medidas de control que pueda implementar la concesionaria para para minimizar las pérdidas en este proceso y en otros similares, involucrando a la Autoridad Aeronáutica Nacional.

\section{Método}

El desarrollo de la investigación combinó datos cualitativos y cuantitativos incorporando la observación directa, las estadísticas disponibles, la recolección de información de informantes clave y el uso de fuentes secundarias.

Los datos de fuentes primarias, algunas de ellas aún no publicadas la facilitó la Concesionaria Interairport, las referencias sobre el conocimiento o desconocimiento de las tarifas se obtuvo por medio de una encuesta aplicada a una muestra de actores relacionados con el proceso de cobros examinado en la investigación.

Se propone una investigación indagatoria, ya que no se conocen otros trabajos con estas mismas características aplicadas al mismo objeto analizado; lo cual puede ser debido a que hasta ahora este tipo de cobros han sido considerados de menor importancia tanto para la Concesionaria como para la Autoridad Aeronáutica. No obstante, también se desarrollan en la indagación, aportes descriptivos sobre todo al momento de explicar el problema abordado y una posible solución. 
La población del estudio son los pilotos de vuelos privados que han aterrizado y despegado en el Aeropuerto Internacional Toncontín. La muestra de pilotos a entrevistar fue definida en relación con la cantidad de vuelos privados registrados por la Concesionaria (Cálculo hecho en base a Vivanco, 2005). La cantidad de vuelos privados registrada en Toncontín, tomando como referencia el semestre que se extiende de septiembre de 2013 hasta febrero 2014, según matrícula de aeronave y que tuviera más de un vuelo registrado, es de 43 , por lo cual se constató que la muestra a tomar con índice de confianza de $95 \%$ y un coeficiente de variación del $5 \%$, debe ser de 39 pilotos. La aplicación de una encuesta en este caso dio el dato del porcentaje de pilotos privados que conocen el procedimiento a seguir al aterrizar y despegar su aeronave en Toncontín, con lo cual se obtuvo un referente de las pérdidas por deficiencia de cobro que estaría absorbiendo tanto la Concesionaria como la Autoridad Aeronáutica.

Los datos estadísticos relevantes para la investigación fueron la facturación de servicios aeroportuarios, los movimientos registrados de vuelos privados, los recibos de pago por servicios privados cancelados y la estadísticas de vuelos privados; de estos datos se obtuvo la frecuencia de los vuelos privado en Toncontín, la comparación entre la facturación emitida versus los recibos de cancelación y la cantidad de vuelos de un piloto privado en el Toncontín.

\section{Resultados}

Se presentan en este acápite tres resultados, los cobros y retenciones que corresponden a la Concesionaria, la ruta crítica que siguen los cobros aeroportuarios de aeronaves privadas y, un resumen de las respuestas obtenidas en la encuesta aplicada.

\section{Tarifas y cobros en Toncontín}

El marco legal del tipo de cobros examinados distingue ente cobros y retenciones. En cuanto a los cobros se establece que la Concesionaria tiene plena capacidad para establecerlos (Interairport, Estado de Honduras, 2000. 3.2.1.) y hacerlos efectivos, con la posibilidad de aplicar penalización de intereses en aquellos casos que tal cosa fuera necesaria. La aplicación de estos cobros seguirán los siguientes principios (Interairport, Estado de Honduras, 2000. 3.2.3.):

- Todos los usuarios deberán pagar por los servicios que el Concesionario preste en los Aeropuertos. Quedan exceptuados aquellos supuestos en que la exención del pago esté reconocida por las prácticas internacionales del sector y 
aquéllos otros supuestos en que la legislación hondureña y/o el presente contrato así lo permita;

- Los operadores de aeronaves que sean usuarios satisfarán su parte correspondiente del costo de suministro de las instalaciones y servicios que están incluidos en las actividades aéreas de los aeropuertos.

- Los cobros de aeropuerto no deben ser de una magnitud tal que se desaliente el uso de las instalaciones y servicios que son necesarios para la seguridad;

- Con carácter general, no se cobrará a los usuarios por instalaciones y servicios que no utilizan.

En cuanto a los cobros se agrega que el Concesionario no establecerá cobros por concepto de cualquier servicio o instalación en importes que perjudiquen el ejercicio de la libre competencia (Interairport, Estado de Honduras, 2000. 3.2.4.).

Por lo que respecta a las retenciones, se establece que la Concesionaria puede solicitar a la Autoridad Aeroportuaria la no autorización de los planes de vuelos de aquellas aeronaves que no hayan hecho el pago por aeropuerto que les corresponde, además el concesionario puede solicitar el embargo de la aeronave de un operador que estuviera en mora o hubiere incumplido sus obligaciones referentes al pago correspondiente (Interairport, Estado de Honduras, 2000. 4.1); se definen como circunstancias de mora las siguientes:

- Para aquellas aerolíneas con las que el Concesionario tenga una relación por virtud de la cual no se les exija el pago del servicio correspondiente inmediatamente después de su uso, cuando vencida la fecha de pago de las tarifas y cobros de aeropuertos debidas por el período correspondiente de liquidación, de acuerdo con los compromisos alcanzados entre el Concesionario y dicho operador de aeronaves, hayan transcurrido más de diez días sin que dicho pago se haya hecho efectivo (Interairport, Estado de Honduras, 2000. 4.1.1.).

- Para aquellos operadores de aeronaves que no estén en la situación descrita, cuando no se efectúe el pago de las tarifas y cobros del aeropuerto correspondiente inmediatamente después de hacerse uso del servicio que la origina (Interairport, Estado de Honduras, 2000. 4.1.2.).

La Autoridad Aeronáutica sólo puede desistir de cumplir con la solicitud del Concesionario en la medida que dichos pagos estén siendo objeto de litigio entre 
dicho operador de aeronaves y el Concesionario o cuando las circunstancias en el momento en cuestión sean de fuerza mayor o emergencia.

El instrumento de consulta fue aplicado a 39 pilotos de los cuales 35 se dedican a vuelos privados, el resto hace otro tipo de vuelos.

\section{Procedimiento de cobro de servicios aeroportuarios}

Se propone un procedimiento definido con la finalidad de asegurar el buen funcionamiento de las operaciones y mejorar los servicios ofrecidos. Todo el proceso de cobro comprende cuatro pasos:

- Cálculo, revisión y cobro de servicios aeroportuarios al contado para los vuelos privados; para este fin los pesos de las aeronaves se obtienen de los certificados de aeronavegabilidad o del manual de mantenimiento que porta el piloto, el cual debe corresponder a la matrícula de la aeronave, caso contrario se tomará como válido el Peso Máximo de Despegue, más alto para el tipo de aeronave en cuestión encontrado en la base de datos del Concesionario. Además se deben hacer cálculos de pesos y balances de las aeronaves, en el caso de facturar el peso se lo presentan en kilos se multiplicará por 2.2 y se lo dividirá entre 2000, para obtener un resultado en toneladas. Ejemplo: kilos 10,000X2=Lb 22,000/2000= Toneladas 11.00. Las tarifas serán pagadas en Dólares o su equivalente en Lempiras calculado el día que se efectúe el pago, al tipo de cambio señalado por el Banco Central de Honduras. Las tarifas establecidas afectan los aterrizajes nacionales (US\$ 2.79 por tonelada, con US\$ 9.29 de facturación mínima) e internacionales (US\$ 1.17, con US\$ 5.80 de facturación mínima), el estacionamiento en rampa nacional (US\$ 0.45 por tonelada) e internacional (US\$ 0.45 por tonelada), la iluminación de pista internacional (US\$ 1.39 por tonelada) y nacional (US\$ 1.39 por tonelada) y, el puente de embarque internacional (US\$ 58.08 por aeronave por cada dos horas o fracción) y nacional (US\$22.62 por aeronave por cada dos horas 0 fracción). En el documento de tarifas se ilustra la forma de cobro en cada caso con ejemplos .

- Facturación del cobro a través del sistema interno de pagos de Interairport (NAF por sus siglas en inglés).

- Elaboración y emisión de recibo de pago. 
- Al final del día hacer entrega del efectivo recibido a la persona encargada de realizar el arqueo de ese día.

\section{Flujograma 1: Proceso de cobro de Servicios Aeroportuarios}

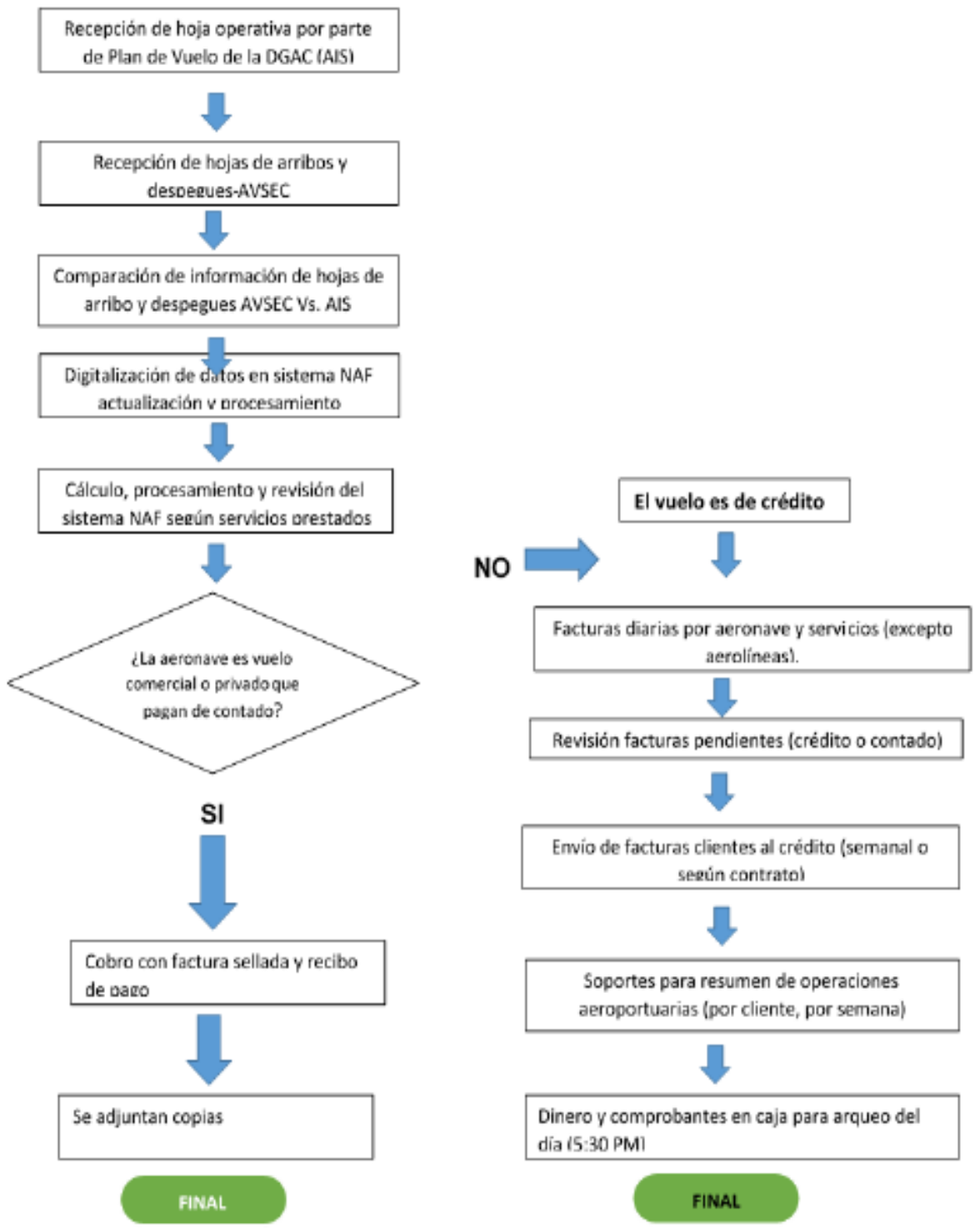


Resultados de la consulta con usuarios

Gráfico 1 ¿Con qué frecuencia ha aterrizado en Toncontín entre enero y marzo de 2014?

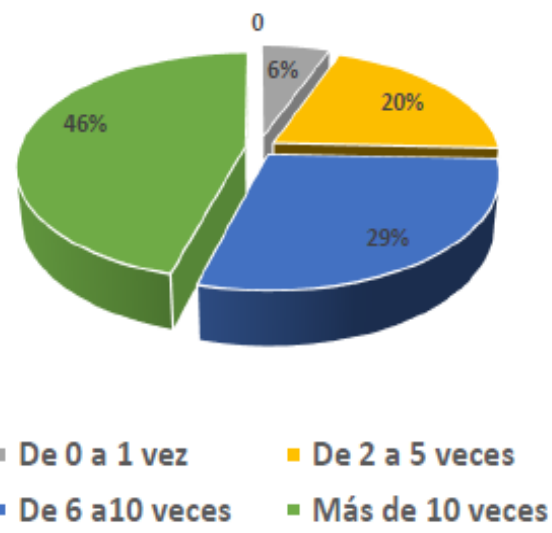

Como se puede ver los entrevistados hacen un uso frecuente del Aeropuerto de Toncontín, con lo cual se puede presumir que conocen el ambiente incluyendo el personal que atiende, los espacios en donde se hacen los pagos, la calidad de los servicios recibidos, los lugares físicos que ocupan en el Aeropuerto las Autoridades Aeronáuticas encargadas de supervisar los cobros, seguramente también la cultura organizacional reflejada en el tipo de atención recibida, entre otros aspectos. Esto aleja la posibilidad del no pago por desconocimiento del entorno de cobro.

Gráfico 2: ¿Conoce si existe un procedimiento a seguir para el pago de los servicios aeroportuarios?

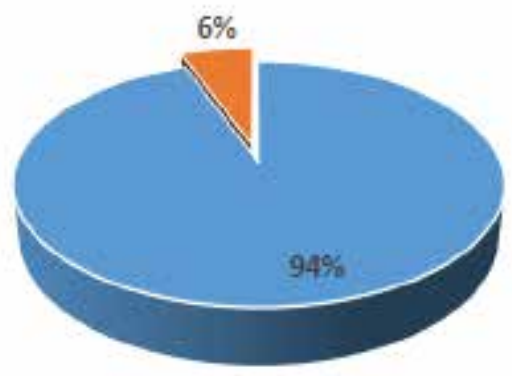


En una entrevista de otro estudio sobre temas aeronáuticos se constata que los técnicos y profesionales dan gran importancia al cumplimiento de la reglamentación aeronáutica y se agrega que en este campo este conocimiento es un imprescindible punto de partida para la formación y la capacitación en el trabajo (Colindres, 2014); casi ningún piloto entrevistado (6\%) demostró ser excepción a esta regla, con lo cual se puede afirmar que el procedimiento de pago es bastante bien conocido.

\section{Gráfico 3: ¿Conoce cuál es la tarifa a pagar por servicios aeroportuarios?}

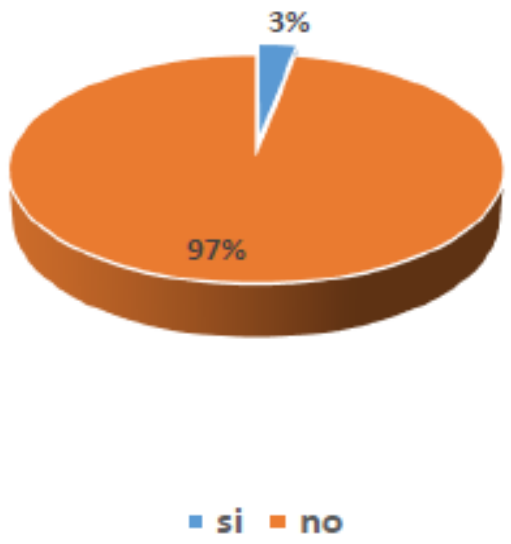

El conocimiento del procedimiento y el saber a quiénes se debe pagar no es suficiente para el pago se haga efectivo, si hubiese pago se sabría también los montos pagados. Si estos porcentajes se invirtieran los pilotos usuarios de servicios estarían en mejores condiciones para establecer una relación costo-beneficio desde la cual se podría incentivar los pagos.

Gráfico 4 ¿Le han explicado el proceso de cobro de servicios de INTERAIRPORT?

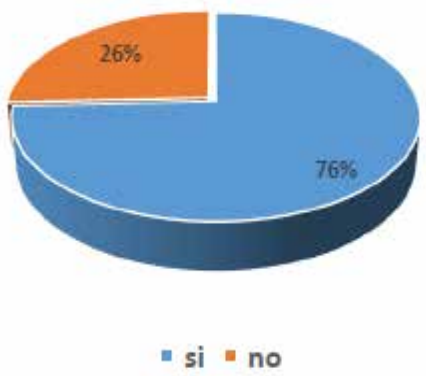


Esta vez las respuestas se presentan mejor repartidas que en los gráficos anteriores, sin embargo es notorio que a un número importante de pilotos, tres tercios del total $(76 \%)$, ya se les ha explicado el proceso de pago de servicios. No se puede inferir de estos resultados que el tercio al que no se ha explicado no conozca el proceso, primero porque ya saben que existe un procedimiento de cobro, segundo, porque los cobros por estos servicios son comunes en cualquier aeropuerto con las características del Toncontín.

Gráfico 6: ¿Le explicaron el proceso a seguir antes de la aprobación del plan de vuelo por parte de la DGAC?

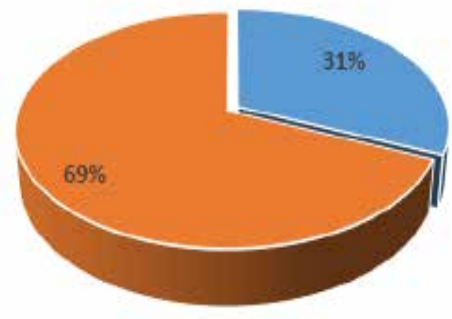

- SI $=$ NO

En este caso se refleja con datos cuantitativos una falencia de la Autoridad Aeronáutica que es a quién le corresponde no sólo explicar el proceso mencionado, sino también condicionar la aprobación del plan de vuelo al cumplimiento de los pagos correspondientes.

Gráfico 7: ¿Cuáles son los mayores problemas para pagar los servicios aeroportuarios?

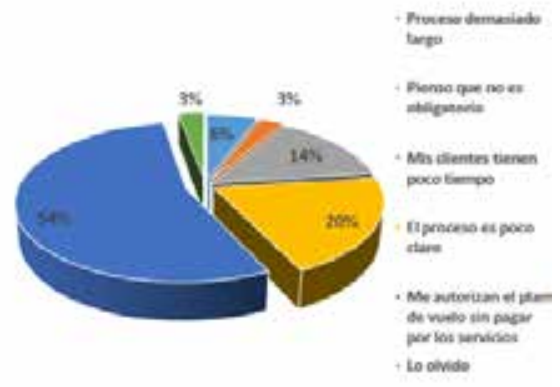


Con la tendencia de esta respuesta se muestra una vez más la ausencia de la Autoridad Aeroportuaria en el proceso de cobro: la autorización del plan de vuelo no está condicionada al pago de los servicios. Se podría concluir que en cualquier caso la pérdida por los cobros no es muy alta o que la absorbe la Concesionaria, pero otro efecto indirecto de esta falta de cobro puede ser la disminución del carácter de Autoridad que asiste a la AHAC en esta o en cualquier otra de sus actividades como representante del Estado de Honduras en el ambiente aeronáutico civil.

\section{Conclusiones}

- De acuerdo a los resultados obtenidos en la investigación se evidencia que la falta de coordinación entre el personal de la concesionaria y el personal de plan de vuelo de la DGAC impide que se cumpla a cabalidad el proceso de cobro por servicios aeroportuarios.

- Existe un procedimiento de cobro por servicios aeroportuarios establecido por la concesionaria, respaldado en el contrato de concesión celebrado entre el concesionario y el Estado de Hondura a través de SOPTRAVI, con lo cual se ofrece una base suficiente para hacer los cobros como aplicación de la regulación vigente.

- La encuesta revela que en su mayoría los pilotos privados conocen el procedimiento, de lo cual se pueden inferir que ambos departamentos tanto el de servicios aeroportuarios de la concesionaria como el de plan de vuelo de la DGAC, han cumplido su labor en el sentido de dar a conocer dicho procedimiento; es evidente entonces que lo que ha faltado ha sido hacer efectivo el cumplimiento de los cobros, aplicando las herramientas que ya ofrece la reglamentación y panificando estratégicamente la recuperación de estos cobros.

- La no cancelación de los valores facturados por servicios aeroportuarios genera pérdidas económicas para el concesionario, así como para el Estado el cual recibe el $34.4 \%$ de todos los ingresos facturados por el concesionario.

- Independientemente del beneficio de recuperación de un valor monetario, la aplicación del procedimiento de cobro por servicios puede ser una oportunidad de trabajo coordinado entre la AHAC y la Concesionaria que se puede aplicar a otros campos de trabajo en donde la coordinación podría reportar beneficios a ambas partes. 


\section{Recomendaciones}

- Programar contactos entre la Jefatura Administrativa del concesionario encargada del Departamento de Servicios Aeroportuarios y el Departamento de Plan de Vuelo de la DGAC para hacer un planteamiento del problema de la falta de cobro y sus repercusiones tanto en los ingresos de la Concesionaria como en la merma de la Autoridad de la Agencia Hondureña de Aeronáutica Civil.

- Solicitar la colaboración de la AHAC mediante la Oficina de Plan de Vuelo en los términos que lo indica el contrato de concesión para la no autorización de los vuelos que no presenten su comprobante de pago por los servicios aeroportuarios prestados por el concesionario.

- Mejorar la comunicación entre el Departamentos de Servicios Aeroportuarios del concesionario y la Oficina de Plan de Vuelo de la AHAC con el fin de que el procedimiento se cumpla con la participación armónica y coordinada de las dos entidades encargadas.

- Emitir una circular por parte de la AHAC ya que es la Autoridad Aeronáutica del País en la que se indique a los pilotos privados que sus planes de vuelo serán autorizados únicamente sin han cumplido con el requisito de cancelar los servicios aeroportuarios prestados por el concesionario.

- Redactar un volante por parte del Concesionario en el que se indique el proceso a seguir para la cancelación de los servicios aeroportuario, resaltando los beneficios de los servicios prestados y la obligatoriedad del pago.

\section{Bibliografía}

- Haslett, C. (2008). Essentials of Radio Wave Propagation. Ofcom, UK: Cambridge University Press.

- Richards, J. A. (2008). Radio Wave Propagation An Introduction for the Non-Specialist. Canberra, Australia: Springer.

- Aeropuertos de Honduras. (2013). Reporte estadístico mensual. Tegucigalpa: No publicado. 
- Aeropuertos de Honduras SA. (2012). Manual de procedimiento de cobro por servicios aeroportuarios. Tegucigalpa, Francisco Morazán , Honduras: No publicado.

- Alberto Colindres, A. M. (2014). Cuerpo de Contenido para la Formación de Controlador de Tránsito Aéreo en el ámbito de las ciencias aeronáuticas en Honduras. Tegucigalpa: No publicado.

- American Association of Variable Star Observers (AAVSO). (14 de Junio de 2012). Sudden Ionispheric Disturbances (SIDs). Recuperado el 08 de 11 de 2012, de American Association of Variable Star Observers (AAVSO): http:// www.aavso.org/solar-sids

- American Meteorological Society. (2012). Glossary of meteorology. Allen Press.

- Castillo, Y. (2012). Diseño de un radiotelescopio para actividades de investigación . Revista de Ciencias espaciales, --.

- Chernan, C. (1978). The handbook of solar flare monitoring \& propagation forecasting. Blue rdge, USA: Tab Books.

- Committee on the Societal and Economic Impacts of Severe Space Weather Events. (2008). Severe Space Weather Events - Understanding Societal and Events: A Workshop. Washington, DC, United States of America: The National academy Press.

- Cummer, S., Inan, U., \& Bell, T. (Noviembre-Diciembre de 1998). lonospheric $\mathrm{D}$ region remote sensing using VLF radio atmpspherics. Radio Science, 33(6), 1781-1792.

- Department of the Navy Naval Electronic Systems Command. (1972). Naval Shore Electronic Criteria: VLF, LF, and MF Comunication Systems. Washington: U.S. Printing Office.

- DGAC. (2004). Ley de Aeronáutica Civil. Tegucigalpa: DGAC.

- DGAC Chile. (22 de Noviembre de 2012). DGAC. Obtenido de www.dgac.gob. cl/portal 
- Dixon, B. (1991). " La Arquitectura del periodo Formativo y la competencia del estado social en Yarumela, Honduras. En YAXKIN. Honduras .

- Dixon, B. (2008). Yarumela: una historia de investigacion arqueologica en el sitio y su lugar en la antigua historia hondureña. En R. YAXKIN, Revista del instituto hondureño de antropologia e historia (Vol. XXIV, pág. 199). Tegucigalpa, Honduras: ENAG.

- Embry Riddle. (2012). Undergraduate/Graduate Catalog. Daytona: Embry Riddle.

- FUMEC. (27 de Noviembre de 2012). Universidade FUMEC. Obtenido de www. fumec.br/cursos

- Grubor, D., Suli, D., \& Zigman, V. (2005). Influence Of Solar X-Ray Flares On The Earth-lonosphere Waveguide. Serbian Astronomical Jounal, 29-35.

- Haridas, D., Soman, K. P., \& Sundaram, S. (2013). Ionospheric Disturbances due to Solar lonospheric Disturbances due to Solar. International Journal of Advanced Electrical and Electronics Engineering, 69-74.

- Hunsucker, R. D., \& Hargreaves, J. K. (2003). The high-latitude ionosphere and its effects on radio propagation. Cambridge, UK: Cambridge University Press.

- Interairport, Estado de Honduras. (23 de septiembre de 2000). Contrato de concesiones. Tegucigalpa, Honduras: La Gaceta.

- J.S. Perez, E. M. (1999). Manual Sanitario para Tripulantes de Cabina de Pasajeros. Madrid: Arán Editores.

- Kolarski, A., Grubor, D., \& Suli, D. (8 de Agosto de 2011). Diagnostics Of The Solar X-Flare Impact On Lower lonosphere Through The VIf-Naa Signal Recordings. Baltic Astronomy, 20, 591-595.

- Lang, J.-P. (10 de 05 de 2013). GNU Radio. Obtenido de Welcome to GNU Radio!: http://gnuradio.org/redmine/projects/gnuradio/wiki

- Lashley, J. (2010). The Radio Sky and How to Observe It. (M. Inglis, Ed.) New York, USA: Springer. 
- Leech, M. (28 de 03 de 2014). Proyecto SIDSuite. Obtenido de The Comprehensive GNU Radio Archive Network: https://www.cgran.org/browser/projects/ SIDSuite.

- Mandeville, J. (1986). "Proyecto Arqueologico Valle de Comayagua: Investigaciones en Yarumela-Chical. IX N² 2,1986, 17-41.

- Mandeville, J. (1997). "Programa Arqueologico de Yarumela, temporada 1983: descubrimientos y analisis". XV, edicion extraordinaria 1990-1994, 5-18.

- More, C., Sharma, A., Bhonsle, R., \& Lynn, K. (2010). Fiel Strength Measurement of VLF Waveguide of VLF RadioWave Propagation at $19.8 \mathrm{KHz}$ betwen Australia and India. Australian Space Science Conferene Series (págs. 249262). Sydney: Wain.

- Nakamura, S. (1991). Desarrrollo y decaimiento en la periferia de Copán". annals of Latin American studies, 39-95.

- National Bureau of Standards. (1937). Radio Instruments and Measurements. Washington: GOVERNMENT PRINTING OFFICE.

- NOAA/Space Weather Prediction Center. (8 de Octubre de 2012). Space Weather Prediction Center. Recuperado el 27 de Octubre de 2012, de http://www. swpc.noaa.gov/SolarCycle/index.html

- OACI. (2006). Doc 9562 Manual sobre los aspectos económicos de los aeropuertos, Segunda Edición. Montreal: $\mathrm{OACl}$.

- OACI. (2011). Estudio de la OACI revela gran demanda de personal aeronáutico cualificado de aquí al Año 2030. OACl.

- OACl. (20 de Noviembre de 2012). ICAO. Obtenido de icai.int/safety/dangerous good

- radiosky.com. (30 de 10 de 2013). Radio-SkyPipe II - An Internet Enabled Strip Chart Recorder. Obtenido de Radio-SkyPipe II - An Internet Enabled Strip Chart Recorder: http://www.radiosky.com/skypipeishere.html 
- Raulin, J., Berton, F., Gavilán, H., Guevara, W., Rodriguez, R., Fernandez, G., . . Hadano, R. (2010). Solar flare detection sensitivity using the South America VLF(SAVNET). Journal Of Geophysical Research, 115, A07301.

- Raulin, J., Correia de Matos, P., Hadano, R., Saraiva, A., Correia, E., \& Kaufmann, P. (2009). The Souh America VLF Network (SAVNET):Development installation status, first results. Geofísca Internacional, 48(3), 253-261.

- Sampieri, H. (2010). Metodología de la investigación. México: McGraw Hill.

- spaceweather.com. (s.f.). The Classification of X-ray Solar Flares. Recuperado el Octubre de 2010 de 28, de http://spaceweather.com: http://spaceweather. com/glossary/flareclasses.html

- U S P M. (27 de Noviembre de 2012). Universidad San Martín de Porres. Obtenido de www.usmp.edu.pe/ffia/aeronauticas

- UKRAA. (30 de 10 de 2013). UK Radio Astronomy Association. Obtenido de UK Radio Astronomy Association: http://www.ukraa.com/www/

- Universidad Técnica Federico de Santa María. (27 de Noviembre de 2012). Ingeniería en aviación comercial. Obtenido de www.usm.edu.cl/admision

- USAC. (s.f.). Atlas Arqueologico de Guatemala. (D. G. Natural, Productor) Obtenido de http://www.atlasarqueologico.com/.

- Valladarez, O. A. (Septiembre de 2008). Yarumela: Revalorizacion un sitio aqueológico en el valle de Comayagua. (ENAG, Ed.) YAXKIN, XX1V, No1, 2112226.

- Vivanco, M. (2005). Muestreo estadístico, diseño y aplicaciones. Santiago.

- Wolf, D. (30 de 10 de 2013). Audio Spectrum Analyzer . Obtenido de Audio Spectrum Analyzer : http://www.qsl.net/dl4yhf/spectra1.html 